\title{
Sasang constitution affects the prevalence of functional dyspepsia
}

\author{
Yoon Jeong Kim¹, Yo Chan $\mathrm{Ahn}^{2}$ and Chang Gue Son ${ }^{1 *}$
}

\begin{abstract}
Background: Functional dyspepsia (FD), which is a very common disorder worldwide, is known to be caused by multiple factors including environmental and genetic factors. Sasang constitutional medicine (SCM) is a component of traditional Korean medicine that emphasizes inherited characteristics of the physical and psychological patterns of a patient. This study investigated whether the prevalence of FD differs depending on Sasang classification.

Methods: A total 517 subjects (190 males and 327 females) were recruited, and interviewed for the presence of FD using a Rome III-based questionnaire. The Sasang constitution of all subjects were diagnosed using a Sasang constitutional analytical tool (SCAT). A Chi-square test was performed to compare prevalence of DF among different Sasang constitutional types.
\end{abstract}

Results: Of the 517 subjects, 115 (22.2\%) met the diagnostic criteria for FD, and the prevalence was significantly higher in females $(26.9 \%)$ than males $(14.2 \%, p<0.01)$. The Sasang-constitution-based prevalence among all subjects was $27.5 \%$ for Taeumin, $23.1 \%$ for Soumin, and $16.4 \%$ for Soyangin $(p=0.055)$. When compared by sex, the prevalence of FD among Sasang types showed significantly different patterns between males and females $(p<0.05)$; in females with FD, Taeumin predominated (32.5\% compared with $29.5 \%$ and $18.8 \%$ for Soumin and Soyangin, respectively; $p<0.05$ ), whereas males with FD displayed a higher prevalence of Soumin (17.3\% compared with $9.1 \%$ and $11.3 \%$ for Taeumin and Soyangin, respectively; $p>0.05$ ).

Conclusions: This study identified significant differences in FD prevalence depending on Sasang constitution and sex. Our findings provide data to guide future research on the prevention and management of FD.

Keywords: Functional dyspepsia, Sasang constitution, Traditional Chinese medicine, Traditional Korean medicine

\section{Background}

Functional dyspepsia (FD) is defined as a condition in which dyspeptic symptoms occur in the epigastric region in the absence of an organic disease that explains them [1]. FD is one of the most common medical conditions, with a worldwide prevalence of approximately 11-29.2\% [2]. Symptoms of FD are non-specific, and the disorder seriously impairs patients' daily functioning and quality of life [3].

The pathophysiology of FD is highly diverse, and involves delayed gastric emptying, hypersensitivity to gastric distension, altered gastrointestinal motility and gastric electrical rhythms, and dysregulation of the autonomic nervous-central nervous system relationship $[4,5]$. The

\footnotetext{
*Correspondence: ckson@dju.ac.kr

'Liver and Immunology Research Center, Daejeon Oriental Hospital of Daejeon University, \# 176-9 Daeheung-ro, Jung-gu, Daejeon 302-724, Republic of Korea

Full list of author information is available at the end of the article
}

main pathogenetic factors of FD include genetic predisposition, psychosocial factors, infection from Helicobacter pylori, and inflammation [6, 7]. These multicausal and multifactorial characteristics of FD partially explain why few universally effective therapies exist for the disorder [8]. Accordingly, patients with FD frequently use complementary and alternative medicine, including herbal remedies and acupuncture, as an important choice alongside conventional treatment $[9,10]$.

Sasang constitutional medicine (SCM), a field of traditional Korean medicine (TKM), emphasizes the inherited characteristics of psychological and physical patterns, as well as the combination of congenital determinants and internal organ function in the development of diseases or disorders [11]. Based on these inherited traits, SCM classifies people into four types: Taeyangin, Soyangin, Taeumin, and Soumin [12]. Several studies have proposed constitutional-type- 
specific susceptibility to certain disorders, such as a high prevalence of ischemic stroke in Taeumin and lower digestive function in Soumin subjects [13, 14]. Additionally, a genetic role in the development of FD has been well demonstrated $[15,16]$. However, few studies have investigated the relationships between the development of FD and Sasang constitution classification.

The present study investigated how FD prevalence varies according to Sasang constitution.

\section{Methods}

\section{Subjects}

In total, 517 participants (aged 17-77 years) were recruited among general population in Daejeon city of South Korea, from November, $1^{\text {st }}, 2013$ to December, 31st, 2014 (Table 1). Participants consisted of 190 males and 327 females, and their occupations were varied like college student, office worker, and others. Any subject who had to take a medicine due to a distinct organic disease was excluded from the study. Informed consent was obtained from each subject, and the Ethics Committee of Daejeon University Hospital approved the study protocol (Authorization number: DJOMC-118).

\section{Diagnosis of FD}

Based on a self-reported questionnaire, potential participants were interviewed by a doctor to ascertain the presence of FD according to the Rome III Diagnostic Criteria [17], which are: patient complains of one or more of bothersome postprandial fullness, early satiation, epigastric pain, or epigastric burning lasting at least 3 months with symptom onset at least 3 months prior to diagnosis. Subjects diagnosed with FD did not have any structural disease that was likely to explain their symptoms, as determined by a doctor's interview regarding whether an endoscopy had been performed, physical examination, laboratory (hematological and biochemical) tests, and medical history.

\section{Classification of Sasang constitution}

Each participant was classified as having one of the four types of Sasang constitution (Taeumin, Taeyangin, Soyangin, or Soumin) using the Sasang constitutional analytical tool (SCAT). The SCAT is a web-based Sasang constitution decision-making system that was developed by the
Korea Institute of Oriental Medicine [18]. The selection of Sasang constitution is made by an integrative combination of information from the subject's body shape (10 points), facial contour analysis (10 points), voice features (5 points), along with physiologic symptoms collected by questionnaire (10 points). Once individual levels of all required items were entered into the SCAT system, it presented the percentage of each person's potential to be categorized as a Sasang constitution type. Then, a person's Sasang constitution with the highest percentage is adopted as the person's final type.

\section{Statistical analysis}

Statistical analysis of the data was performed using SAS statistical software (SAS Rel. 8.02; SAS Institute, Inc., Cary, NC, USA). A Pearson's Chi-square test or two-way ANOVA was used to compare the prevalence of FD in terms of Sasang constitution type and sex. $P$-values $<0.05$ were considered statistically significant.

\section{Results}

\section{Prevalence of FD by sex}

Among 517 participants, 115 met the diagnostic criteria for FD, for a total prevalence of $22.2 \%$. The prevalence in males was $14.2 \%$ (27 of 190 subjects); it was significantly higher in females, at $26.9 \%$ (88 of 327 subjects, $p<0.01$, Table 2).

\section{Distribution of Sasang constitutions}

The proportions of Taeumin, Soumin, and Soyangin constitution types among the total participants were $29.6 \%$, $38.5 \%$, and $31.9 \%$, respectively. When the Sasang constitution was analyzed according to sex, male subjects showed prevalence of $17.4 \%, 54.7 \%$, and $27.9 \%$, and female subjects showed prevalence of $36.7 \%, 29.1 \%$, and $34.3 \%$ for Taeumin, Soumin, and Soyangin, respectively. Among the 115 subjects with FD, the Sasang distribution was $36.5 \%$ (11.1 \% in males, $44.3 \%$ in females), $40.0 \%$ (66.7 \% in males, $31.8 \%$ in females), and $23.5 \%$ (22.2 \% in males, $23.9 \%$ in females) for Taeumin, Soumin, and Soyangin, respectively (Table 2).

\section{Prevalence of FD among Sasang constitutions}

The differential distribution of FD prevalence according to Sasang constitution type was: $27.5 \%$ for Taeumin, $23.1 \%$

Table 1 Characteristics of study participants

\begin{tabular}{lll}
\hline Characteristics & Male & Female \\
\hline Number (\%) & $190(36.8)$ & $327(43.2)$ \\
Median age (years, range) & $23(18-64)$ & $21(17-77)$ \\
Median height (cm, range) & $173.5(161.3-189.2)$ & $161.0(142.6-176.0)$ \\
Median weight (kg, range) & $70.2(44.3-96.6)$ & $55.8(40.4-98.0)$ \\
Mean BMI \pm SD & $23.5 \pm 2.8$ & $21.9 \pm 3.5$ \\
\hline
\end{tabular}


Table 2 Distribution of patients with FD according to Sasang classification and sex

\begin{tabular}{lllll}
\hline Subjects (\%) & & Taeumin & Soumin & Soyangin \\
\hline Total subjects & Male: $190(100)$ & $33(17.4)$ & $104(54.7)$ & $53(27.9)$ \\
& Female: $327(100)$ & $120(36.7)$ & $95(29.1)$ & $112(34.3)$ \\
& Total: $517(100)$ & $153(29.6)$ & $199(38.5)$ & $165(31.9)$ \\
Subjects with FD & Male: $27(100)$ & $3(11.1)$ & $18(66.7)$ & $6(22.2)$ \\
& Female: $88(100)$ & $39(44.3)$ & $28(31.8)$ & $21(23.9)$ \\
& Total: $115(100)$ & $42(36.5)$ & $46(40.0)$ & $27(23.5)$ \\
FD prevalence ${ }^{* *}$, \# & ${ }^{a}$ Male: $14.2 \%$ & $9.1 \%$ & $17.3 \%$ & $11.3 \%$ \\
& ${ }^{*}$ Female: $26.9 \%$ & $32.5 \%$ & $29.5 \%$ & $18.8 \%$ \\
& Total: $22.2 \%$ & $27.5 \%$ & $23.1 \%$ & $16.4 \%$ \\
\hline
\end{tabular}

A comparison of FD prevalence between males and females $\left({ }^{* *} p<0.01\right)$ and among Sasang constitution types in male ( $\left.{ }^{\alpha} p=0.389\right)$, female $\left({ }^{*} p<0.05\right)$, and all subjects $\left({ }^{\beta} p=0.055\right)$ obtained by Pearson's Chi-square test. The sex-dependent difference in FD prevalence in terms of Sasang constitution $\left({ }^{\#} p<0.05\right)$ was shown via a two-way ANOVA test

for Soumin, and $16.4 \%$ for Soyangin $(p=0.055)$. When analyzed separately by sex, the prevalence of FD according to Sasang constitution differed significantly according to sex. More women FD prevalence was of the Taeumin type (32.5\% compared with $29.5 \%$ and $18.8 \%$ for Soumin and Soyangin, respectively, $p<0.05)$. The prevalence of FD among men did not differ significantly according to Sasang type $(p=0.389)$, although there was a Soumindominant tendency (17.3\% compared with $9.1 \%$ and $11.3 \%$ for Taeumin and Soyangin, respectively). This sexdependent difference in the pattern of FD prevalence was significant $(p<0.05$, Table 2).

\section{Discussion}

Current genetic science has shown that inherited genomic variance is a critical factor affecting individual differences in the development of various diseases [19]. Genomic or constitutional components are closely related to the occurrence of FD [20, 21]. SCM emphasizes the importance of the inherited characteristics of psychological and physical patterns in the development of disease and in therapeutic responses [22]. Accordingly, it is assumed that each type of Sasang constitution shows a different susceptibility to FD.

Our results reveal that $22.2 \%$ of the total subject was belonged in FD. This result is similar to the data from a Korean nationwide survey showing a $20.4 \%$ prevalence [23]. The prevalence of FD has varied considerably depending on the population or definition used. The $14.7 \%$, $17 \%$, and $23.8 \%$ prevalence were reported from studies in Norway [24], Japan [25], and the US [26], respectively. The presence of true FD, where organic disease is excluded, is difficult to determine in population studies due to logistic difficulties [2].

SCM classifies people into four constitutional types based on the weighted Yin-Yang balance and the functional imbalance between two internal organs; Taeyangin (greater Yang) individuals are characterized as having excessive lung function and deficient liver function, whereas Taeumin (greater $\mathrm{Yin}$ ) individuals show the opposite pattern. Soyangin (lesser Yang) individuals have excessive spleen function and deficient kidney function, whereas Soumin (lesser Yin) individuals show the opposite pattern [27]. It is generally understood that the Taeyangin type is extremely rare in the Korean population [28], and none of the 517 study subjects in the present study was classified as Taeyangin.

Despite a general belief that the prevalence of FD is relatively equal in males and females, two studies, from Taiwan and Australia, showed significantly higher FD prevalence in females compared to males [29, 30]. Both the previous Korean study [23] and the present study found a higher prevalence of FD in females by approximately 2 -folds ( $14.2 \%$ of male versus $26.9 \%$ in females). When the FD prevalence was investigated according to Sasang constitution, Taeumin $(27.5 \%)$ and Soumin $(23.1 \%)$ individuals displayed notably higher FD prevalence compared with Soyangin (16.4\%) subjects, a difference that approached statistical significance $(p=$ 0.055). Our results also found especially a Sasang-typedependent difference in susceptibility to FD, even within the same sex. We observed a Taeumin-prevalent pattern in females versus a Soumin-prevalent pattern in males. Our data may suppose the highest susceptibility of FD in Taeumin female versus the lowest FD susceptibility in Taeumin male, as 3.6-fold higher in male comparing to female. This finding may indicate the different manners of affections by Sasang constitutional characteristics between males and females. Sex-related differences in the development of various diseases are well known [31]. Our previous study found a significant difference in cancer prevalence according to Sasang classification and gender, with higher cancer prevalence in Soumin-type males and Soyangin-type females [32]. These findings would recommend the Sasang constitution as an important medicine in diagnosis or therapeutics of FD.

Recently, several studies have reported that subjects of certain Sasang classifications are notably more susceptible to some disorders; e.g., Taeumin for type 2 diabetes and metabolic syndrome [33, 34] and Soumin for irritable bowel syndrome [35]. We are unable to explain the mechanisms responsible for the association between Sasang constitution and the tendency to develop particular disorders, including FD. However, there are some clues to support the clinical phenomena. SCM classification is assumed to be connected to macro-level genomic differences [36, 37]. Biological and psychological characteristics including diet habits are associated with Sasang constitutional types [38, 39]. Interestingly, one study proposed a possible link between Sasang type and gut microbial environment [40]. Psychological characteristics, 
dietary habits, and gut flora are all known as factors that strongly affect the development of FD [41].

FD is a very common disorder that reduces health-related quality of life and impairs vitality. However, currently, no standard effective therapy exists due to the multicausal and multifactorial characteristics of the disorder [42]. Our findings demonstrate firstly an association between Sasang constitution and FD prevalence. Our study however had limitations, such as the relatively small number of subjects from a single city in Korea. We therefore have to pay careful attention in interpretation of our finding, which should be confirmed by a large scale of study in the future. We also need the further studies explaining the corresponding mechanisms of differences in FD prevalence among Sasang constitutional types, for example regarding any patterns of genomic differences, psychological characteristics, diet habits, and gut microbial environment.

\section{Conclusion}

In conclusion, our results identified a significantly different pattern in FD prevalence among Sasang constitutions, and this pattern differed by sex. These findings will benefit future research on the prevention and management of FD.

\section{Competing interest}

The authors declare that there is no conflict of interests regarding the publication of this manuscript.

\section{Author contribution}

YJ carried out the clinical studies, coordinated all processes, and YC performed statistical analysis. CG mainly participated in the design and conduction of the study. All authors read and approved the final manuscript.

\section{Author information}

CG Son is a professor of Daejeon Oriental Hospital of Daejeon University, and a director of Liver and Immunology Research Center. YJ Kim is a researching nurse for clinical study in Daejeon Oriental Hospital of Daejeon University. YC Ahn is a professor of department of health service management in Daejeon University.

\section{Acknowledgments}

This work was supported by a National Research Foundation of Korea (NRF) grant funded by the Ministry of Science, ICT \& Future Planning (No. 2006-2005173).

\section{Author details}

${ }^{1}$ Liver and Immunology Research Center, Daejeon Oriental Hospital of Daejeon University, \# 176-9 Daeheung-ro, Jung-gu, Daejeon 302-724, Republic of Korea. ${ }^{2}$ Department of Health Service Management, Daejeon University, \# 62 Daehak-ro Dong-gu, Daejeon 300-716, Republic of Korea.

Received: 25 February 2015 Accepted: 13 May 2015 Published online: 20 May 2015

\section{References}

1. Miwa H. Why dyspepsia can occur without organic disease: pathogenesis and management of functional dyspepsia. J Gastroenterol. 2012;47:862-71.

2. Mahadeva S, Goh KL. Epidemiology of functional dyspepsia: a global perspective. World J Gastroenterol. 2006;12:2661-6.

3. El-Serag HB, Talley NJ. Health-related quality of life in functional dyspepsia. Aliment Pharmacol Ther. 2003;18:387-93.

4. Oustamanolakis P, Tack J. Dyspepsia: organic versus functional. Clin Gastroenterol. 2012;46:175-90.
5. O'Mahony S, Dinan TG, Keeling PW, Chua AS. Central serotonergic and noradrenergic receptors in functional dyspepsia. World J Gastroenterol. 2006;12:2681-7.

6. Kim SE, Park YS, Kim N, Kim MS, Jo HJ, Shin CM, et al. Effect of Helicobacter pylori eradication on functional dyspepsia. J Neurogastroenterol Motil. 2013;19:233-43.

7. Allescher HD. Functional dyspepsia-a multicausal disease and its therapy. Phytomedicine. 2006;13:2-11.

8. Madisch A, Miehlke S, Labenz J. Management of functional dyspepsia: unsolved problems and new perspectives. World J Gastroenterol. 2005;11:6577-81

9. Suzuki H, Inadomi JM, Hibi T. Japanese herbal medicine in functional gastrointestinal disorders. Neurogastroenterol Motil. 2009;21:688-96.

10. Zeng F, Qin W, Ma T, Sun J, Tang Y, Yuan K, et al. Influence of acupuncture treatment on cerebral activity in functional dyspepsia patients and its relationship with efficacy. Am J Gastroenterol. 2012;107:1236-47.

11. Chae H, Lyoo IK, Lee SJ, Cho S, Bae H, Hong M, et al. An alternative way to individualized medicine: psychological and physical traits of Sasang typology. J Altern Complement Med. 2003;9:519-28.

12. Lee JM. Longevity and life preservation in oriental medicine. In: Choi SH, editor. Kyung Hee University Press, Seoul, South Korea; 1996.

13. Lee BC, Doo HK, Ahn SY, Byun SH, Kim SI, Park HK, et al. Peroxisome proliferator-activated receptor-gamma Pro12Ala polymorphism is associated with the susceptibility to ischemic stroke in Taeeumin classified by Sasang medicine. Neurol Res. 2007;29:S32-7.

14. Lee MS, Sohn KW, Kim YH, Hwang MW, Kwon YK, Bae NY, et al. Digestive system related pathophysiological symptoms of Sasang typology: systematic review. Integrative Medicine Research. 2013;2:39-48.

15. van Lelyveld N, Linde JT, Schipper M, Samsom M. Candidate genotypes associated with functional dyspepsia. Neurogastroenterol Motil. 2008;20:767-73.

16. Arisawa T, Tahara T, Shiroeda H, Minato T, Matsue $Y$, Saito T, et al. Genetic polymorphisms of SCN10A are associated with functional dyspepsia in Japanese subjects. J Gastroenterol. 2013;48:73-80.

17. Song KH, Jung HK, Min BH, Youn YH, Choi KD, Keum BR, et al. Development and validation of the Korean Rome III questionnaire for diagnosis of functional gastrointestinal disorders. J Neurogastroenterol Motil. 2013;19:509-15.

18. Do JH, Jang E, Ku B, Jang JS, Kim H, Kim JY. Development of an integrated Sasang constitution diagnosis method using face, body shape, voice, and questionnaire information. BMC Complement Altern Med. 2012;12:85.

19. Gabriel S. Variation in the human genome and the inherited basis of common disease. Semin Oncol. 2006;33:S46-9.

20. Oshima T, Toyoshima F, Nakajima S, Fukui H, Watari J, Miwa H. Genetic factors for functional dyspepsia. J Gastroenterol Hepatol. 2011;26:83-7.

21. Santonicola A, Siniscalchi M, Capone P, Gallotta S, Ciacci C, lovino P. Prevalence of functional dyspepsia and its subgroups in patients with eating disorders. World J Gastroenterol. 2012;18:4379-85.

22. Yoo J1, Lee E, Kim C, Lee J, Lixing L. Sasang constitutional medicine and traditional chinese medicine: a comparative overview. Evid Based Complement Alternat Med 2012; 2012:980807.

23. Kim SE, Park HK, Kim N, Joo YE, Baik GH, Shin JE, et al. Prevalence and risk factors of functional dyspepsia: a nationwide multicenter prospective study in Korea. J Clin Gastroenterol. 2014;48:e12-8.

24. Bernersen B, Johnsen R, Straume B. Non-ulcer dyspepsia and peptic ulcer: the distribution in a population and their relation to risk factors. Gut. 1996;38:822-5.

25. Hirakawa K, Adachi K, Amano K, Katsube T, Ishihara S, Fukuda R, et al. Prevalence of non-ulcer dyspepsia in the Japanese population. J Gastroenterol Hepatol. 1999;14:1083-7.

26. Shaib Y, El-Serag HB. The prevalence and risk factors of functional dyspepsia in a multiethnic population in the United States. Am J Gastroenterol. 2004;99:2210-6.

27. Song IB, Koh BH, Lee EJ. Sasang Constitutional Medicine. $2^{\text {nd }}$ (in Korean). Jipmoondang, Seoul, South Korea, 2004

28. Lee TG, Hwang MW, Ham TI, Choi BK, Koh BH, Song IB. A study on distributional rate of Sasangin in Korea. J Sasang Constitution Medicine. 2005;17:12-21.

29. Lu CL, Lang HC, Chang FY, Chen CY, Luo JC, Wang SS, et al. Prevalence and health/social impacts of functional dyspepsia in Taiwan: a study based on the Rome criteria questionnaire survey assisted by endoscopic exclusion among a physical check-up population. Scand J Gastroenterol. 2005;40:402-11. 
30. Koloski NA, Talley NJ, Boyce PM. Epidemiology and health care seeking in the functional Gl disorders: a population-based study. Am J Gastroenterol. 2002;97:2290-9.

31. Benigni R. Social sexual inequality and sex difference in cancer incidence. Environ Res. 2007;104:128-34

32. Lee J, Kang W, Cho J, Cho C, Yoo H, Son C. Cancer incidence varies significantly depending on sasang constitution of traditional Korean medicine. J Tradit Chin Med. 2013;33:312-5.

33. Cho NH, Kim JY, Kim SS, Lee SK, Shin C. Predicting type 2 diabetes using Sasang constitutional medicine. J Diabetes Investig. 2014;5:525-32.

34. Jang E, Baek Y, Park K, Lee S. The sasang constitution as an independent risk factor for metabolic syndrome: propensity matching analysis. Evid Based Complement Alternat Med. 2013:2013:492941.

35. Lee SK, Yoon DW, Yi H, Lee SW, Kim JY, Kim JK, et al. So-eum type as an independent risk factor for irritable bowel syndrome: a population-based study in Korea. J Altern Complement Med. 2014;20:846-52.

36. Kim BY, Jin HJ, Kim JY. Genome-wide association analysis of Sasang constitution in the Korean population. J Altern Complement Med. 2012;18:262-9.

37. Kim BY, Yu SG, Kim JY, Song KH. Pathways involved in sasang constitution from genome-wide analysis in a Korean population. J Altern Complement Med. 2012;18:1070-80

38. Lee SJ, Park SH, Cloninger CR, Kim YH, Hwang M, Chae H. Biopsychological traits of Sasang typology based on Sasang personality questionnaire and body mass index. BMC Complement Altern Med. 2014;14:315.

39. Pham DD, Lee JC, Lee MS, Kim JY. Sasang types may differ in eating rate, meal size, and regular appetite: a systematic literature review. Asia Pac J Clin Nutr. 2012;21:327-37.

40. Kim BS, Bae HS, Lim CY, Kim MJ, Seo JG, Kim JY, et al. Comparison of gut microbiota between Sasang constitutions. Evid Based Complement Alternat Med. 2013:2013:171643.

41. Van Oudenhove L, Aziz Q. The role of psychosocial factors and psychiatric disorders in functional dyspepsia. Nat Rev Gastroenterol Hepatol. 2013;10:158-67.

42. Tack J, Masaoka T, Janssen P. Functional dyspepsia. Curr Opin Gastroenterol. 2011;27:549-57.

\section{Submit your next manuscript to BioMed Central and take full advantage of:}

- Convenient online submission

- Thorough peer review

- No space constraints or color figure charges

- Immediate publication on acceptance

- Inclusion in PubMed, CAS, Scopus and Google Scholar

- Research which is freely available for redistribution 